\title{
Research on the Construction of Multi-dimensional Power Grid Enterprise Asset Health Evaluation Index System
}

\author{
CHENG Jia xu*1,a, WANG Guan $\operatorname{ran}^{2}$, LIU Jin ${ }^{3}$ \\ ${ }^{1}$ State Grid Energy research Institute Co., LTD Beijing, China \\ ${ }^{2}$ North China Electric Power University Beijing, China \\ ${ }^{3}$ State Grid Energy research Institute Co., LTD Beijing, China
}

\begin{abstract}
Most power grid companies are asset-intensive and technology-intensive companies. Therefore, the quality and health of assets and equipment are crucial to the sustainability of the development of power grid companies and the safety of power grid development. To power grid companies through multiple dimensions Decompose the evaluation index of asset health, find the key factors affecting the asset health evaluation of power grid enterprises, and find a way to solve the problem for the subsequent improvement of the health of power grid enterprises' assets. This paper describes the SMART criteria for asset health assessment, combining the principles of index selection and index grading, and clarifies the principles of constructing the evaluation index system. On this basis, from the three dimensions of physical, economic and social, and considering the internal and external power grid enterprises, a multi-dimensional asset health evaluation index system has been established.
\end{abstract}

\section{Introduction}

Most power grid companies are asset-intensive and technology-intensive companies. Therefore, the quality and health of assets and equipment are crucial to the sustainability of the development of power grid companies and the safety of power grid development. To power grid companies through multiple dimensions Decompose the evaluation index of asset health, find the key factors affecting the asset health evaluation of power grid enterprises, and find a way to solve the problem for the subsequent improvement of the health of power grid enterprises' assets.

\section{Principles of Constructing Evaluation Index System}

\subsection{SMART criterion}

The assessment of asset health status is of great value to asset management. To evaluate asset status, it is necessary to follow SMART guidelines, index selection principles, and index grading principles to build a scientific and reasonable asset health evaluation index system. ${ }^{[1]}$

The SMART criterion is a design criterion for the evaluation index system commonly adopted by the World Bank and national government departments. The criterion is composed of the initials of five words, namely Specific, Measurable, Attainable, Relevant, and Trackable.

(1) Specific
The index system is an objective description of the essential characteristics, composition structure and constituent elements of the evaluation object, and serves a specific evaluation activity. For the purpose of evaluation work, the index system should be specific and specialized, mainly manifested as target-specific and orientation-

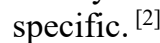

(2) Measurable

The measurability of evaluation indicators means that there should be corresponding standards for the evaluation of indicators, and the same standards shall be used as a unified scale to measure the performance of the evaluated objects. Measurability is not only a requirement for quantitative indicators, but for qualitative indicators, as long as detailed evaluation standards are established, they also need to meet the requirements for measurability. ${ }^{[3]}$

(3) Attainable

The design of the indicator system should take into account the possibility of obtaining the data required for verification. If the data used for the evaluation of an indicator is impossible or difficult to obtain in reality, then the operability of the indicator is questionable. Therefore, attainable means that when designing the index system, it is necessary to consider the way and channels for obtaining evaluation data. ${ }^{[4]}$

(4) Relevant

Each indicator of the evaluation indicator system needs to have a certain degree of relevance, that is, not a stack of multiple indicators, but a set of indicators that are organically connected to each other, and there is a certain internal logical relationship between the indicators. ${ }^{[5]}$

(5) Trackable

$\mathrm{a}^{*}$ Corresponding author shsrp@126.com 
The purpose of evaluation is to monitor. General evaluation activities can be divided into pre-, in-process and post-evaluation. No matter which type of evaluation, the evaluation effect needs to be tracked and re-evaluated after a certain stage. Therefore, when designing evaluation indicators, we should consider whether the corresponding indicators are easy to track, monitor and control. ${ }^{[6]}$

\subsection{Index selection principle}

Indicators reflect the quantitative concept and specific values of system elements or phenomena, including two parts: the name of the indicator and the value of the indicator. The evaluation of asset health status involves many factors. For this reason, it is necessary to combine multiple internally linked indicators according to a certain structural level to form an indicator system. Combined with the characteristics and connotation of asset health evaluation, the selection of indicators should follow seven principles including comprehensiveness, as shown in Figure 1:

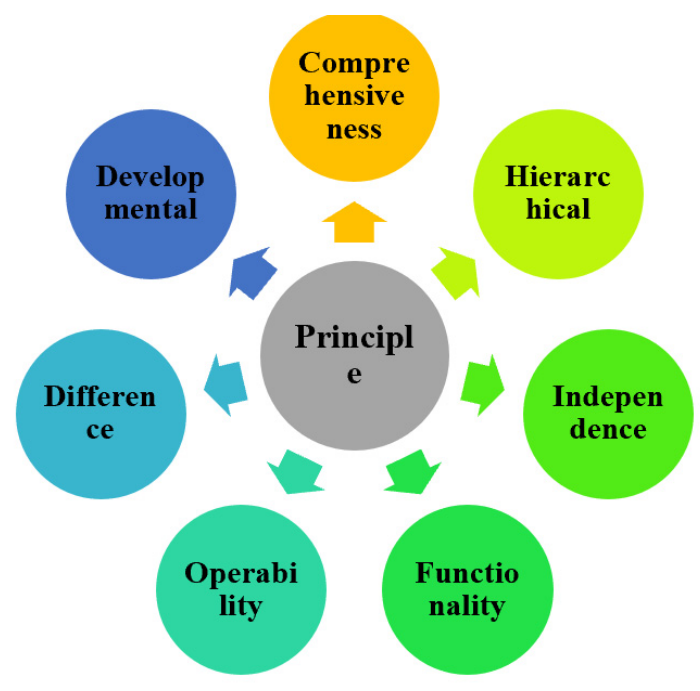

Fig 1. Principles for index selection

(1) The principle of comprehensiveness. As an organic whole, the indicator system should cover all aspects of asset health evaluation, and comprehensively reflect the factors that affect asset health. ${ }^{[7]}$

(2) The principle of hierarchy. Asset health evaluation is a complex system, and the system needs to consider the hierarchy, that is, the combination of indicators should have a certain hierarchical structure.

(3) The principle of independence. There should be a certain internal connection between the indicators, and the correlation and overlap of information should be removed as much as possible, that is, there is a strong correlation between indicators at different levels, and there is no duplication of information between indicators at the same level.

(4) Functional principle. According to the purpose of the research, the functions of indicators can be roughly summarized as: description function, interpretation function, evaluation function, monitoring function, early warning function and decision-making function, improvement function, and promotion function. The functional index system used here can illustrate the gaps in the behavior of each subject for comparison.

(5) The principle of operability. The principle of operability mainly refers to the availability of data. The establishment of the index system should take into account the quantification of the index and the availability, reliability and conciseness of relevant data.

(6) The principle of difference. The evaluation research of asset health status should take into account the differences of regional economic level, social environment and natural environment. The assessment of asset health in each region is carried out to varying degrees. At the same time, the organizational structure of each regional power grid has its own characteristics. It is necessary to fully understand the particularity of the power grid industry in which it is built.

(7) The principle of development. The construction of the indicator system is to better evaluate the overall health of current asset management. The construction of the indicator system should follow the developmental nature. In different development periods, the transfer of the focus of asset management needs to be considered, and the indicator system should be targeted. perfect.

\subsection{Principles of Index Classification}

(1) In order to further screen out the key factors of the evaluation objects and fully grasp the influence of different evaluation indicators on the evaluation objects, the evaluation indicators need to be classified and graded to build a hierarchical index system, as shown in Figure 2.

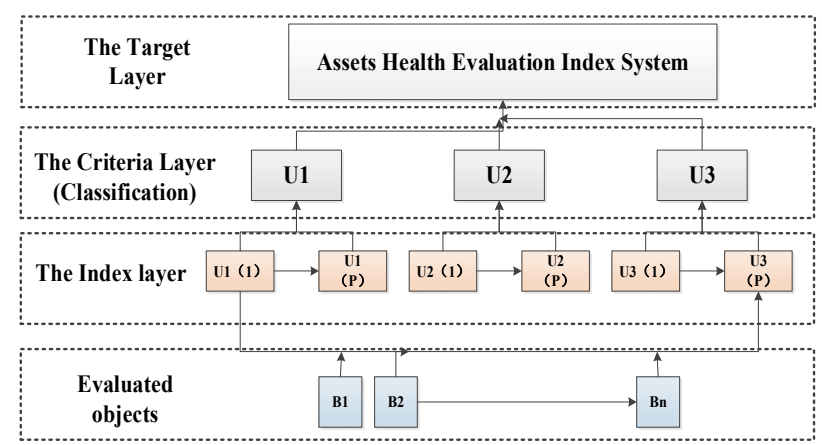

Fig 2. Classification structure of asset health evaluation index system

(2) For the indicator system in Figure 2, each type of indicator is composed of several indicators. Similar indicators have some similarities, while different indicators have different effects on the overall goal, so they cannot be treated equally. An index classification layer is added between the target layer and the index layer to make the hierarchical structure of the index system clearer and more conducive to the analysis of problems. ${ }^{[8]}$

\section{Construction of asset health evaluation index system}

\subsection{Purpose of asset health evaluation}


Asset health evaluation is an important part of asset management. Asset health of power grid is directly related to equipment status. The health of power grid assets refers to the extent to which power equipment can meet the requirements of safe and stable operation of power grid in a certain period of time under the action of reasonable internal and external factors. The purpose of asset health evaluation is to master the health of assets, eliminate the potential faults of power system, prevent the occurrence of sudden accidents, and reduce accident losses. Due to the various types of state information of power equipment, all kinds of information can reflect the operation of power grid from different aspects and levels. It is necessary to process the state information and reasonably select relevant indicators to comprehensively evaluate the health of assets.

According to VBM theory, combined with the principle of index selection and index stratification, considering the value driving factors of asset health, this paper constructs the evaluation index system of asset management health from the physical, economic and social aspects.

\subsection{Physical evaluation index system}

The physical dimension mainly analyzes the health of assets from the perspective of the physical characteristics of power grid. When the score of physical index is low, it indicates that the health of assets is abnormal. It is necessary to determine the specific risk factors according to the three-level index, and make maintenance or replacement equipment decisions. According to the value driving factors, physical property can be carried out from three aspects: asset allocation, asset operation and asset maintenance.

\section{(1)Asset allocation}

The asset allocation status of power grid enterprises is the basis of evaluating the health of assets and reflects the objective equipment situation of the enterprise. Among them, the capacity load ratio and annual load rate reflect the capacity and average load state of the main network; the proportion of intelligent substation, distribution automation and circuit breaker combination rate reflect the intelligence of power grid assets.

(2)Asset operation status

The operation status of power grid enterprises assets is the key to evaluate the health of assets, reflecting the actual operation status of power grid assets. Among them, the average operation life of equipment and the rate of scrapped assets reflect the service life of assets; the comprehensive line loss rate reflects the loss of transmission and distribution lines, and the high line loss rate is the early warning of line failure.

(3)Asset maintenance status

Asset maintenance status is an important guarantee for power grid assets to keep healthy. Maintenance record is an objective record for relevant personnel to maintain power grid equipment. Among them, the average annual maintenance times of equipment reflects the daily inspection of power grid assets; the abnormal state rate of relay protection equipment directly reflects the health status of power grid assets in daily inspection work.
According to the above analysis, the evaluation index system of asset health under the physical dimension is shown in Figure 3.

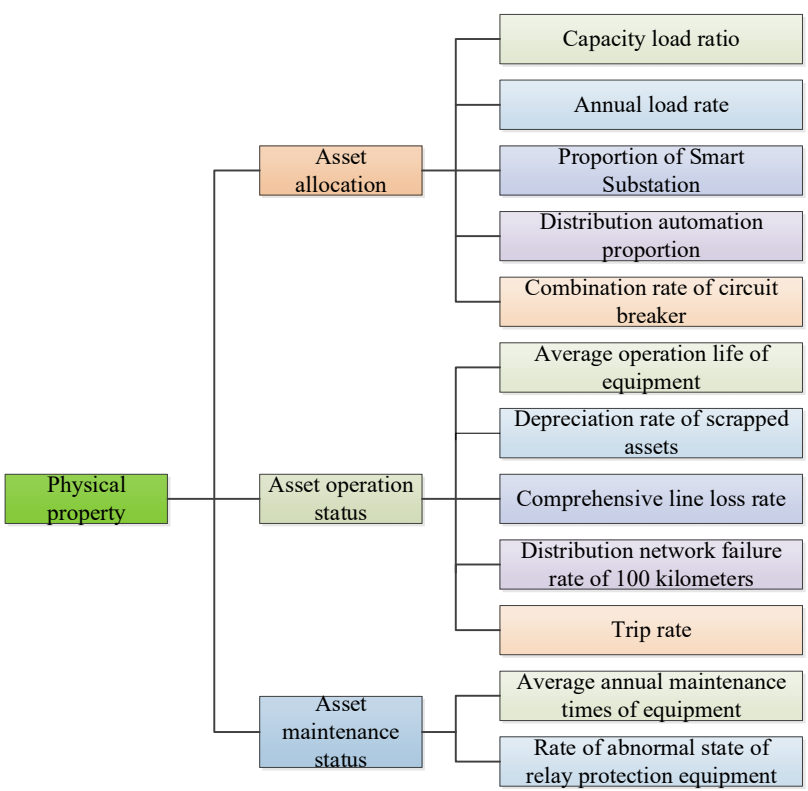

Fig 3. Evaluation index system of asset health in physical dimension

\subsection{Economic Evaluation Index System}

The economic dimension mainly considers the input of grid assets and the corresponding costs incurred. When the physical performance of an asset is better, but the economy is weak, the health of the asset also deviates from the normal level. The economy of assets determines the investment direction of power grid assets to a certain extent. When an asset needs major repair in terms of physical characteristics, but the cost of operation and maintenance is high, decision makers may judge the health state of the asset as abnormal according to the actual situation, and treat the asset as minor repair or scrap. According to the value driving factors, economy can be developed from three levels: asset cost, asset quality and asset efficiency.

(1) Asset cost

Asset cost is the manpower and financial investment of power grid companies to ensure the stable and normal operation of assets. Among them, operation and maintenance costs and asset disposal costs reflect the basic economic expenditures to ensure the health of assets; unit capacity transformation costs and unit capacity transmission costs reflect the preliminary planning and construction expenditures of power grid assets.

(2) Asset quality

Asset quality mainly starts from the economic point of view, and objectively evaluates the overall health of power grid enterprise assets. Among them, the input-output ratio and the income from the sale of electricity per unit of assets reflect the relationship between the profitability of the asset and the cost; the power outage loss of the power grid reflects the direct loss caused by the quality of the asset; the residual value rate of the obsolete asset reflects the value of the asset's recovery and disposal; The annual 
capital investment per ten thousand yuan of assets reflects the annual increase in investment demand for assets.

(3) Asset efficiency

Asset efficiency is composed of basic financial indicators, reflecting the profitability and solvency of assets, including total asset turnover, current asset turnover, fixed asset turnover, total asset return, assetliability ratio, and asset disposal yield.

According to the analysis of the above situation, the evaluation index system of asset health in the economic dimension is shown in Figure 4.

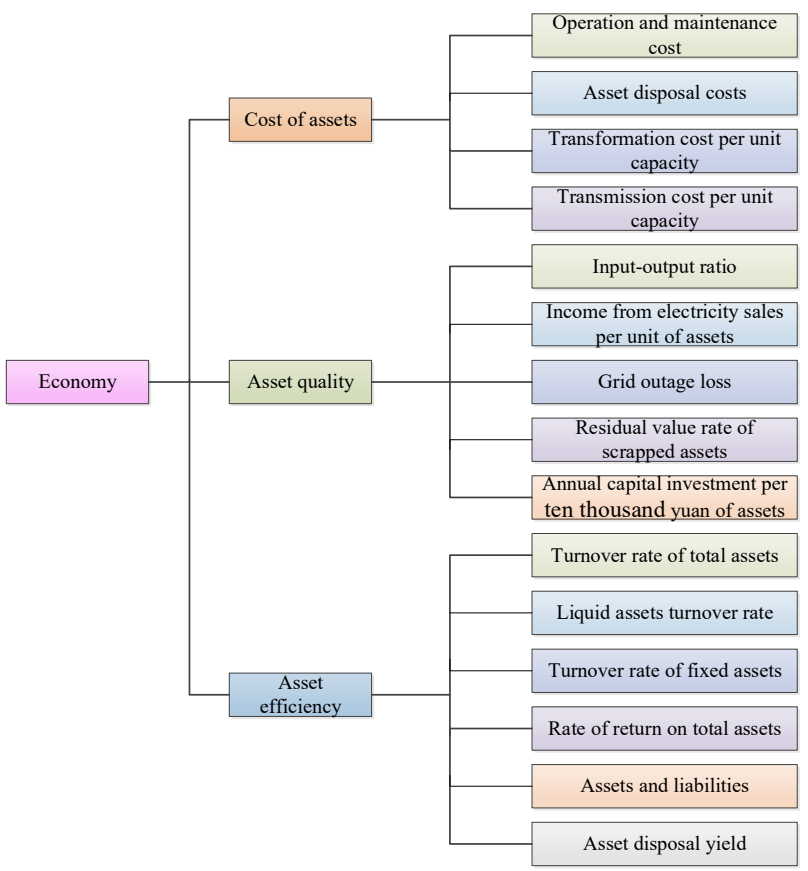

Fig 4. Evaluation index system of asset health in economic dimension

\subsection{Social evaluation index system}

The social dimension is to analyze the asset health from the external perspective of the power grid enterprise. Due to the public nature of electricity, when power grid assets, especially key power equipment, are abnormal, it will have a negative impact on the production and life of the society and users. The healthy social nature of power grid assets mainly considers users' electricity demand, and provides users with reliable and high-quality power supply services. Therefore, the social dimension is an effective supplement to the evaluation of the internal assets of the power grid. According to the value driving factors, sociality can be developed from three levels of power reliability, power safety and user satisfaction.

(1) Electric energy reliability

Satisfying the needs of power users and providing users with reliable power supply services are the basic responsibilities of power grid companies. Power reliability evaluates the health of assets from the basic physical state of power system operation. Among them, the voltage qualification rate and the three-phase current imbalance degree reflect the basic physical properties of the power system; the power supply reliability rate and the average power outage duration reflect the stability of the electric energy from the power outage time level; the spinning reserve rate reflects the power system standby situation. It is an important guarantee for the reliability and stability of the system.

(2) Electric energy safety

Power safety is the most basic requirement for power grid companies to provide power supply services. Two indicators, the number of annual accidents and the coverage rate of relay protection devices, are mainly selected to reflect the security of power grid assets.

(3) User satisfaction

The degree of user satisfaction with the power services provided by the grid company can indirectly reflect the health of the grid company's assets. Among them, the user complaint rate and user power outage losses directly reflect the user's perception of power services; the electricity medical examination saves user costs and reflects the direct value of the health of power grid assets to users.

According to the analysis of the above situation, the evaluation index system of asset health under the social dimension is shown in Figure 5.

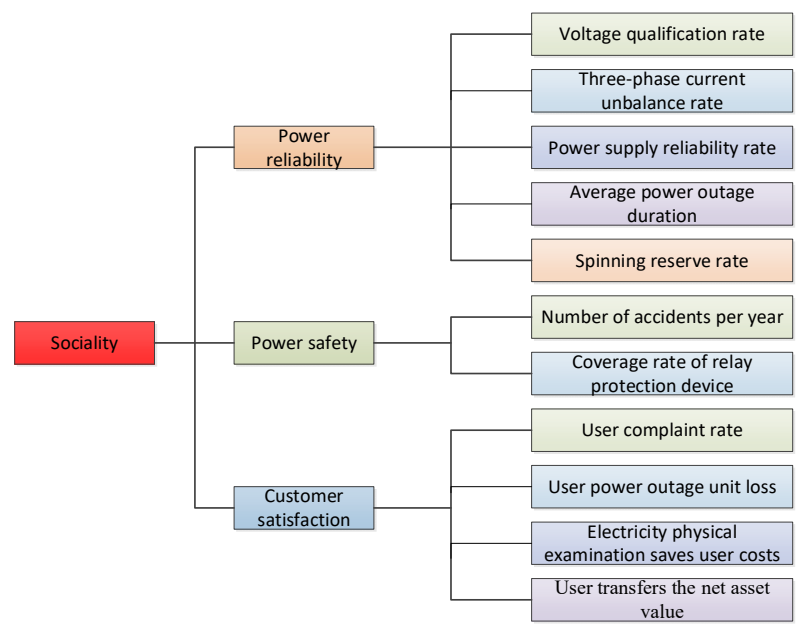

Fig 5. Social dimension asset health evaluation index system

\section{Conclusion}

Combined with physical, economic and social dimensions, we can get a multi-dimensional asset health evaluation index system. Among them, the physical dimension is the most intuitive indicator to reflect the health of assets, and the social dimension indirectly reflects the health of assets.

Considering the health of assets from the internal perspective of power grid enterprises is mainly to evaluate the health of assets from the perspectives of capacity, operation status, fault level, capital cost and capital quality of power equipment in a certain region, which involves two evaluation dimensions of physical and economic. From the external consideration of power grid enterprises, the impact of power grid asset health on society and enterprises is mainly evaluated from the satisfaction of users, which is the social evaluation dimension.

According to the constructed asset health evaluation index system, when evaluating asset health, grid companies need to comprehensively evaluate their health 
from both internal and external perspectives. For example, when an asset or an asset in a certain area shows good internal health, but does not bring due social value, consider analyzing the cause of the abnormal asset status from the user's perspective to improve the service level.

\section{References}

1. Wei Pu, Li Xuyang, Liu Qiming. Construction of comprehensive evaluation index system for analyzing physical assets of power grid [J]. Green technology, 2020 (24): 42-44.

2. Fuyan Liu,Yang Xiaoyong,Yu Min,Shen Jie,Shi Jianfeng,Chen Chao. Research on Diagnosis and Evaluation of Physical Assets of Power Grid Enterprises Based on Combined Weight TOPSIS Theory[J]. IOP Conference Series: Earth and Environmental Science,2020,617(1).

3. Ren Tengyun, Cao Zhen, Chen Gang, Wang Chunbo. Performance evaluation of power grid District asset group based on five dimensional balanced scorecard [J]. Friends of accounting, 2019 (19): 97-102.

4. Hong-xian ZHANG,Zhe WEI,Song XUE, Shi-yu MENG. Research on the Evaluation Model of Investment Economic Benefit of Incremental Power Distribution Project Under the Mode of Power Grid Effective Asset Supervision[A]. Science And Engineering Research Center.Proceedings of 2016 International Conference on Electrical Engineering and Automation ( ICEEA2016) [C].Science And Engineering Research Center:Science and Engineering Research Center, 2016:4.

5. Bai Yu. Research on multidimensional evaluation system of asset life cycle management [J]. China power enterprise management, 2019 (14): 40-41.

6. Yi Xin Sun,Chao Kong,Shuai Yuan,Gang Liu. Research on Analysis and Evaluation System of Power Grid Enterprises Asset Quality[J]. Advanced Materials Research,2014,2912.

7. Chen Ran. Performance evaluation of $Z$ power grid project asset management based on life cycle theory [D]. Beijing Jiaotong University, 2020.

8. Lin Yajing. Research on Comprehensive Plan Management Model of Power Grid Enterprise Based on Key Indicators [J]. China Electric Power Education,2009(23):247-249. 